\title{
Crystallite Sizes and Lattice Distortions of Gel-Spun Ultra-High Molecular Weight Polyethylene Fibers
}

\author{
Xiao-Ping Hu and You-Lo HsIEH \\ Fiber and Polymer Science, Division of Textiles and Clothing, University of California, \\ Davis, CA 95616, U.S.A. \\ (Received October 14, 1997)
}

\begin{abstract}
A detailed method is reported for deriving the lattice distortions and crystallite sizes of ultra-high molecular weight polyethylene (UHMWPE) fibers employing a full wide angle X-ray diffraction (WAXD) pattern. The predominant crystals are orthorhombic form and the refined unit cell dimensions for $a$ and $b$ are $7.40( \pm 0.03)$ and $4.94( \pm 0.03) \AA$, respectively. The average crystallite size normal to 110 planes was found to be in the range of $163-182 \AA$. The microstrain lattice distortion was predominant in the gel-spun UHMWPE fibers, with the lattice constant variation of $0.5 \%$.

KEY WORDS Ultra-High Molecular Weight Polyethylene / Wide Angle X-Ray Diffraction / Paracrystalline Structure / Lattice Distortion / Crystallite Size / Microstrain /
\end{abstract}

The gel-spun ultra-high molecular weight polyethylene (UHMWPE) fibers have superior mechanical properties with the Young's modulus and tensile strength in the range of $100-220 \mathrm{GPa}$ and $3-6 \mathrm{GPa}$, respectively. ${ }^{1}$ Such superior mechanical properties are ascribed to the highly oriented and fully extended chains in the highly crystalline structure. The amount of non-crystalline domains is small, containing mainly chain entanglements, taut tie molecules, and chain ends. ${ }^{2}$

The structure of UHMWPE fibers has been extensively studied. ${ }^{1-20}$ Under ambient conditions, UHMWPE fibers mainly exhibit an orthorhombic crystalline structure with low levels of non-orthorhombic crystals. ${ }^{2-4,13}$ Structural transformations have been observed on UHMWPE fibers. Tension along the fiber axis and lateral compression have shown to cause crystal transformation from the orthorhombic to the monoclinic form. ${ }^{6,10} \mathrm{At}$ either high pressure or the temperature close to its melting point, the orthorhombic crystals go through a solid-state phase transformation to the pseudohexagonal crystals. ${ }^{3,4,10}$

The crystallite sizes and lattice distortions in UHMWPE fibers are not as well understood. The wide angle X-ray diffraction (WAXD) peak widths are known to be closely associated with crystallite sizes. The analysis of peak width is, however, cumbersome owing to the scattering contributions of the amorphous halo and the overlapping of multiple reflections. Valid measurements of the crystallite sizes can only be made when peak overlaps can be properly resolved and separated from the background scatter. Determination of background scattering is the most difficult task and the methods chosen can lead to different results. For instance, crystallite sizes and lattice distortion of the same UHMWPE fibers were different depending upon whether a straight-line background ${ }^{6}$ or the Fourier transform background ${ }^{13}$ was used. Before the line broadening is computed, corrections for air scattering, white light radiation, sample absorption, Lorentz and polarization, and Compton factors have to be made. Likewise the instrumental broadening which arises from the finite width of the slits, thickness of the sample, divergence of the incident beam and the spectral distribution of energy in the incident radiation has to be eliminated.

The crystallite sizes and lattice distortion are usually determined by either integral breadth method or Fourier transform methods. ${ }^{21}$ In the discussion of linear polyethylene crystallized in diluted solution, Hosemann et $a .^{22}$ used the integral breadth method to show that the line profiles were either Lorentz or Lorentz squared functions and the paracrystalline distortions were predominant. The crystallite sizes for UHMWPE fibers were obtained with the assumption that the broadening due to size and the distribution of the disorientation was Gaussian, ${ }^{6,13}$ which may not be always true. The distribution profile can affect the integral breadth, defined as the total area of a reflection divided by the peak maximum intensity, and thus the crystallite size evaluations.

This paper details the use of a full wide angle X-ray diffraction pattern and the integral breadth method to describe the lattice distortion of UHMWPE fibers. The instrumental broadening and the line broadening profiles are considered and the background scattering is represented by a polynomial. Moreover, the discussion about the diffraction trace, peak assignments, and unit cell dimensions are also included.

\section{EXPERIMENTAL}

The gel-spun UHMWPE fibers studied here were Spectra 1000 filaments supplied by Allied Signal, Inc. The fibers were produced by gel-spinning, with a density of $0.964 \mathrm{~g} \mathrm{ml}^{-1}$ and the filament diameter of $28 \mu \mathrm{m}$. The fiber tensile strength was about $3.9 \mathrm{GPa}$ and the elongation to break was about $2.7 \%$. These UHMWPE fibers had a high tensile modulus of $179 \mathrm{GPa} .^{13,18}$

A Scintag XDS 2000 X-ray diffractometer was employed in this work. The $\mathrm{Cu}-K_{\alpha}$ radiation was generated at an accelerating potential of $45 \mathrm{kV}$ and a tube current of $40 \mathrm{~mA}$. The $K_{\beta}$ radiation was filtered by using a single channel analyzer. The X-ray scanning was performed in a reflection mode at the ambient temperature. The sample with about $2 \mathrm{~mm}$ thickness Spectra 1000 fibers was wounded on a zero-background sample holder. Scanning was performed on the equator in the $2 \theta$ ranges 


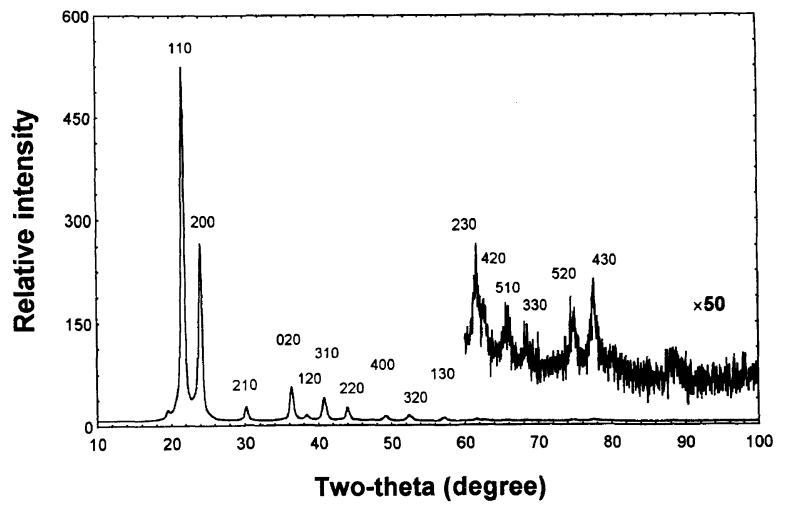

Figure 1. Normalized full equatorial wide angle X-ray diffraction pattern of the spectra 1000 fibers.

of $10^{\circ}-100^{\circ}$. Diffraction intensities were counted at $0.04^{\circ}$-steps and the scanning time of each step was $2.5 \mathrm{~s}$. After data collection, the $\mathrm{Cu}-K_{\alpha 2}$ radiation was stripped by DMS software and output data were further analyzed.

\section{RESULTS AND DISCUSSION}

The data were first corrected, normalized and then resolved by employing the $\mathrm{X}$-ray multi-peak resolution method. ${ }^{23}$ The corrections prior to the peak resolutions were made for air-scattering, white background radiation, polarization and Lorentz factor as well as incoherent scattering (Compton scattering). The intensity reduction affected by specimen absorption was not considered. The corrected intensities were normalized into electron units, and then resolved in terms of peak height $\left(A_{\mathrm{t}}\right)$, peak width at half maximum amplitude $\left(W_{\mathrm{t}}\right)$, peak position $\left(P_{\mathrm{t}}\right)$ and profile function parameter $\left(f_{\mathrm{t}}\right)$ with a mixture form of Gaussian and Lorentz functions. The profile function parameter, $f_{\mathrm{t}}$, defines the form of the peak and varies from 0 to $1\left(f_{\mathrm{t}}=1\right.$ for pure Gaussian and $f_{\mathrm{t}}=0$ for pure Lorentz).

Figure 1 shows the normalized equatorial diffraction pattern of the UHMWPE fibers in the $2 \theta$ range of $10-100^{\circ}$. The most intense peaks were located at 21.61 and $24.02^{\circ}$, corresponding to the 110 and 200 reflections in the orthorhombic form, respectively. The other peaks were relatively weak, and therefore those diffraction trace in the range of $60-100^{\circ}$ were amplified by 50 . Table I summarizes the resolved parameters of the observed peaks and their assignments in an orthorhombic crystal form. A relatively weak peak at $19.63^{\circ}$ is believed to be from the reflection of some form of non-orthorhombic crystals. This peak was first reported to be the 010 reflection in a triclinic form, ${ }^{3,5}$ and later interpreted to be the 010 reflection in a monoclinic form. ${ }^{4,8,11,12}$

The average unit cell dimensions $a$ and $b$ of the orthorhombic crystals were estimated by the full X-ray equatorial pattern and refined by employing the leastsquare between the observed and calculated pattern using two-dimension Fourier transforms ${ }^{24}$ (Table II). The obtained unit cell dimensions were $7.40 \pm 0.03 \AA$ and $4.94 \pm 0.03 \AA$ for $a$ and $b$ direction, respectively. The results are consistent with the structure model proposed by $\mathrm{Fu}$ et $a l .{ }^{13}$ These unit cell dimensions are also close to those in the melt crystallized polyethylene. ${ }^{25}$ Assuming that the unit length along the chain is $2.55 \AA$, the crystal
Table I. Resolved parameters for the equatorial peaks of the Spectra 1000 fibers: Miller indices $(h k l)$, peak position

$(P)$, peak width $(W)$, peak amplitude $(A)$, and profile parameter $(f, f=1$ for Gaussian and $f=0$ for Lorentz)

\begin{tabular}{|c|c|c|c|c|}
\hline$h k l$ & $f$ & $\frac{P}{\operatorname{deg}}$ & $A$ & $\frac{W}{\operatorname{deg}}$ \\
\hline 110 & 0.43 & 21.61 & 529.7 & 0.56 \\
\hline 200 & 0.27 & 24.02 & 258.7 & 0.58 \\
\hline 210 & 0.34 & 30.16 & 19.4 & 0.58 \\
\hline 020 & 0.45 & 36.34 & 48.3 & 0.65 \\
\hline 120 & 0.11 & 38.38 & 6.4 & 0.64 \\
\hline 310 & 0.40 & 40.85 & 32.8 & 0.69 \\
\hline 220 & 0.19 & 44.03 & 18.75 & 0.59 \\
\hline 400 & 0.46 & 49.21 & 6.8 & 0.90 \\
\hline 320 & 0.84 & 52.48 & 7.2 & 1.08 \\
\hline 130 & 0.35 & 57.24 & 5.0 & 0.93 \\
\hline 230 & 0.24 & 61.60 & 2.4 & 0.88 \\
\hline 420 & 0.98 & 62.71 & 1.0 & 0.85 \\
\hline 510 & 1.00 & 65.82 & 0.92 & 1.26 \\
\hline 330 & 0.58 & 68.39 & 0.62 & 0.71 \\
\hline 520 & 0.85 & 74.75 & 1.16 & 1.30 \\
\hline 430 & 0.00 & 77.55 & 2.16 & 0.93 \\
\hline
\end{tabular}

Table II. The observed and calculated equatorial peak $d$-spacings in an orthorhombic crystal $(a=7.40$ and $b=4.94 \AA$ ) for the Spectra 1000 fibers

\begin{tabular}{lll}
\hline$h k l$ & $\frac{d_{\text {obsd. }}}{2}$ & $\frac{d_{\text {cal. }}}{\AA}$ \\
\cline { 2 - 3 } & $\AA$ & $\AA$ \\
\hline 110 & 4.11 & 4.11 \\
200 & 3.70 & 3.70 \\
020 & 2.96 & 2.96 \\
120 & 2.47 & 2.47 \\
310 & 3.43 & 2.34 \\
220 & 2.21 & 2.21 \\
400 & 2.06 & 2.05 \\
320 & 1.85 & 1.85 \\
130 & 1.74 & 1.75 \\
230 & 1.61 & 1.61 \\
420 & 1.50 & 1.50 \\
510 & 1.48 & 1.48 \\
330 & 1.42 & 1.42 \\
520 & 1.37 & 1.37 \\
430 & 1.27 & 1.27 \\
& 1.23 & 1.23 \\
\hline
\end{tabular}

density is then calculated to be $1.00 \mathrm{~g} \mathrm{ml}^{-1}$.

The measurement of the average crystallite size normal to $(h k l)$ planes is usually based upon peak broadening and requires an estimation of the integral breadth of a peak; while the evaluation based on the peak width at half-maximum amplitude does not include the shape factor of the broadening line profile. Valid measurements of the crystallite sizes can only be made when the shape of the line profile is considered and the overlapping peaks in each spectrum have been resolved and separated from the background scatter. As mentioned earlier, determination of background scattering is the most difficult task. The treatments using a straight-line ${ }^{6}$ and Fourier transform $^{13}$ to represent the background scattering have shown to give significantly different crystallite sizes. In this work, we used multi-peaks to represent crystalline structure and a polynomial to represent the amorphous and white background scattering. ${ }^{23}$ 
Table III. The obtained peak width after instrumental broadening correction $\left(W_{2}\right)$ integral breadth $(I B)$, and the apparent crystallite sizes corresponding to those peak widths of un-corrected half-width $\left(\left\langle L_{1}\right\rangle\right)$, corrected half-width $\left(\left\langle L_{2}\right\rangle\right)$ and corrected integral breadth $\left(<L_{3}>\right)$ for Spectra 1000 fibers

\begin{tabular}{|c|c|c|c|c|c|}
\hline \multirow{2}{*}{$h k l$} & $W_{2}$ & $I B$ & $\left\langle L_{1}\right\rangle$ & $\left\langle L_{2}\right\rangle$ & $\left\langle L_{3}\right\rangle$ \\
\hline & deg & $\operatorname{deg}$ & $\AA$ & $\AA$ & $\AA$ \\
\hline 110 & 0.53 & 0.62 & 137 & 150 & 146 \\
\hline 200 & 0.54 & 0.74 & 133 & 148 & 123 \\
\hline 210 & 0.52 & 0.71 & 138 & 154 & 129 \\
\hline 220 & 0.53 & 0.78 & 140 & 158 & 122 \\
\hline 400 & 0.88 & 1.14 & 93 & 98 & 85 \\
\hline 420 & 0.83 & 1.01 & 105 & 110 & 102 \\
\hline 330 & 0.64 & 0.92 & 134 & 147 & 116 \\
\hline
\end{tabular}

Furthermore, the resolved peak broadening, $\mathrm{Br}$, can be caused by structural broadening, $\delta \beta$, as well as instrumental broadening $b r$. The instrumental broadening arises from the finite width of the slits, thickness of the sample, divergence of the incident beam and the spectral distribution of energy in the incident radiation. Many methods have been developed for the evaluation of the instrumental broadening. ${ }^{21}$ In this work, the Fourier-Stokes's method ${ }^{26}$ was employed to separate the instrumental broadening $(b r)$ from the observed and resolved peak broadening $(B r)$, since this method is essentially a deconvolution method and does not depend on the profile functions. The instrumental broadening function was determined from a reference diffraction line of a standard specimen which gives no broadening due to crystal size alone. The main peak at $2 \theta=17.83^{\circ}$ from hexamethylene tetraamine crystals dried overnight at $85^{\circ} \mathrm{C}$ has been used as our standard. ${ }^{24}$

Table III summarizes the full peak width at half amplitude after the instrumental broadening has been eliminated $\left(W_{2}\right)$ and the corresponding integral breadths $(I B)$. Their apparent crystallite sizes corresponded to those peak widths of uncorrected half-height width, corrected half-height width, and corrected integral breadth are represented by $\left\langle L_{1}\right\rangle,\left\langle L_{2}\right\rangle,\left\langle L_{3}\right\rangle$, respectively. These apparent crystallite sizes are also summarized in Table III. Significant differences have been found between $W$ (Table I) and $W_{2}$ (Table III). The instrumental broadening was found to be around $10 \%$ of the total structural broadening, depending upon the line profile shape and the $2 \theta$ position of a reflection. Therefore, the instrumental broadening should not be ignored in the discussion of crystallite sizes. Moreover, the significant differences between the full peak widths and integral breadths have also been detected, indicating that the shape factor of a line profile plays an important role on the calculations of crystallite dimensions.

The structural factors include the crystallite size, size distribution and crystal lattice distortions. For a crystal without any lattice distortion, the integral breadth of a resolved peak is just determined by the average crystalline size.

$$
\delta \beta(c)=\frac{K \lambda}{\left\langle L_{h k l}\right\rangle \cos \theta}
$$

Where $\theta$ is the Bragg angle for the reflection, $\lambda$ is the

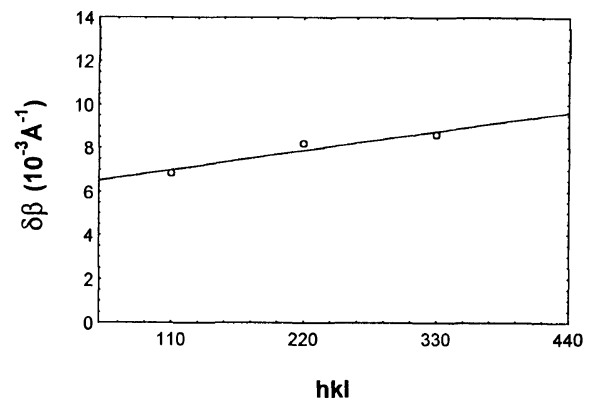

Figure 2. Integral breadth versus reflection-order $(h)$ for the spectra 1000 fibers.

wavelength of the radiation, $\delta \beta(c)$ is the integral breadth of the reflection in radian after the correction for the instrumental broadening, $K$ is the Scherrer parameter which is related to the orientation and peak profile function (usually taken as 1 for fibers ${ }^{25}$ ), and $\left\langle L_{h k l}\right\rangle$ is the average crystallite size normal to the $(h k l)$ planes. For convenience, the above formula can be expressed as below:

$$
\delta \beta(c) \cong \frac{1}{\left\langle L_{h k l}\right\rangle}
$$

For crystals having lattice distortions, additional broadening due to two principle types of lattice distortions of microstrain and paracrystalline structure presented in polymer crystals also appear. Before computing the crystallite sizes, hence, these contributions have to be subtracted. If microstrains are present, the integral breadth of the additional broadening in the reflection is described as:

$$
\delta \beta(S t)=\frac{\sqrt{2 \pi\left\langle\varepsilon_{h k l}^{2}\right\rangle}}{\left\langle d_{h k l}\right\rangle} h
$$

where $h$ is the reflection-order and the $\varepsilon_{h k l}=\Delta d_{h k l} / d_{h k l}$ is a measure of the relative lattice constant variation. In case of paracrystalline distortions the additional line broadening is given as:

$$
\delta \beta(P c)=\frac{1}{\left\langle d_{h k l}\right\rangle}\left(\pi g_{h k l} h\right)^{2}
$$

where $\left(g_{h k l}\right)^{2}=\Delta^{2} d_{h k l} /\left\langle d_{h k l}\right\rangle^{2}$ is the variation between neighboring $(h k l)$ planes and $\Delta^{2} d_{h k l}=\left\langle d_{h k l}^{2}\right\rangle-\left\langle d_{h k l}\right\rangle^{2}$. Thus the quantity $g_{h k l}$ is the reduced variance of the interplanar separation vector.

Figure 2 shows the plot of $\delta \beta$ versus $h$ for the 110 , 220, 330 reflections of Spectra 1000 fibers. The $\delta \beta$ and $h$ seemed to be linearly related, fluctuating around the following straight line:

$$
\delta \beta=6.126+0.88 h
$$

with correlation coefficient being 0.96 . However, it is worthwhile to note that the resolved parameters for peak 110 and 220 are more reliable than those for peak 330 , due to the very intensive peak 110 and 220 and weak peak 330. A peak with weak intensity causes the poor peak resolution and often lead to sharper peak width result. In this case, the minimum peak width for peak 330 should be $9.54 \times 10^{-3} \AA^{-1}$ and thus the crystallite size normal to 110 planes was about $163-182 \AA$. This 
dimension was calculated from extrapolation of the plot to $h=0$ since $\delta \beta(c)$ does not depend on the reflectionorder $h$. These results are in good agreement with other data reported on gel-spun polyethylene fibers. ${ }^{2,5,6,13,18,19}$ However, they are much smaller than those for the single crystals and melt crystallized linear polyethylene. The crystallite dimensions for the single crystals and melt crystallized and annealed linear polyethylene normal to 110 planes were reported to be in the range of 285 $736 \AA .^{2,5,6,13,18,19}$

The microstrain lattice distortion seemed predominantly present in UHMWPE fibers, owing to the generally linear relationship between $\delta \beta$ and $h$. For the single crystals and melt crystallized linear polyethylene, however, paracrystalline distortion was found to be predominant. ${ }^{21,22}$ The reason is probably that there are not only small crystallite sizes and wide crystallite size distribution presented in gel-spun UHMWPE fibers, but also an intermediate phase which could give line broadening exists; whereas in single crystal polyethylene there are usually paracrystalline distortion governing the structure. The observation of dominant microstrain fibers is consistent with the results in Fu et al.'s ${ }^{13}$ work. The estimated parameter for the relative lattice constant variation is approximately $\langle\varepsilon\rangle=0.5 \%$. By analyzing the plot and dependence, therefore, the desired information about the crystallite sizes and the type of lattice distribution can be obtained.

By using the same methods, the crystallite dimensions normal to 200 and 210 were found to be 216 and 293 , respectively, presuming the microstrain distortion is predominant. If the paracrystalline structure and microstrains are present, the mathematical summation of the peak broadening contributed by them would be more complicated, as it is shown in ref 21 .

\section{CONCLUSIONS}

This paper presented a detailed method for deriving the lattice distortions and crystallite sizes of UHMWPE fibers. A full WAXD pattern of the UHMWPE fibers was obtained and the integral breadth method was used to describe the lattice distortions of UHMWPE fibers. The predominant crystals are orthorhombic form and the refined $a$ and $b$ unit cell dimensions are $7.40 \pm 0.03 \AA$ and $4.94 \pm 0.03 \AA$, respectively. The average crystallite size normal to 110 planes was in the range of $163-182 \AA$.
It was also found that the microstrain lattice distortion was predominantly presented in the gel-spun UHMWPE fibers, with the lattice constant variation being approximately $0.5 \%$.

\section{REFERENCES}

1. J. A. H. M. Moonen, W. A. C. Roovers, R. J. Meier, and B. J. Kip, J. Polym. Sci., Polym. Phys. Ed., 40, 361 (1992).

2. Y.-L. Hsieh and X.-P. Hu, J. Polym. Sci., Polym. Phys. Ed., 35, 263 (1997).

3. A. J. Pennings and A. Zwijnenburg, J. Polym. Sci., Polym. Phys. Ed., 17, 1011 (1979).

4. K. Kaji, Y. Ohta, H. Yasuda, and M. Murano, Polym. J., 22, 455 (1990).

5. H. D. Chanzy, P. Smith, J.-F. Revol, and R. St. John Manley, Polym. Commun., 28, 133 (1987).

6. W. R. Busing, Macromolecules, 23, 4608 (1990).

7. K. Tanaka, T. Seto, and T. Hara, J. Phys. Soc. Jpn., 17, 873 (1962).

8. W. Chen, Y. Fu, B. Wunderlich, and J. Cheng, J. Polym. Sci., Polym. Phys. Ed., 32, 2661 (1994).

9. K. Kaji, Y. Ohta, H. Yasuda, and M. Murano, Polym. J., 22, 893 (1990).

10. Y.-L. Hsieh and J. Ju, J. Appl. Polym. Sci., 53, 347 (1994).

11. S. Tsubakihara and M. Yasuniwa, Polym. J., 28, 563 (1996).

12. S. Tsubakihara, A. Nakamura, and M. Yasuniwa, Polym. J., 28, 489 (1996).

13. Y. Fu, W. Chen, M. Pyda, D. Londono, B. Annis, A. Boller, A. Habenschuss, J. Cheng, and B. Wunderlish, J. Macromol. Sci-Phys., B35, 37 (1996).

14. M. Goldman, R. Gronsky, R. Ranganathan, and L. Pruitt, Polymer, 37, 2909 (1996).

15. L. Govaert, B. Brown, and P. Smith, Macromolecules, 25, 3480 (1992).

16. J. Smook and A. J. Pennings, Colloid Polym. Sci., 262, 712 (1984).

17. A. Zwijnenburg, P. E. van Hutten, A. J. Pennings, and H. D. Chanzy, Colloid Polym. Sci., 256, 729 (1978).

18. N. Khosravi, S. B. Warner, N. S. Murthy, and S. Kumar, J. Appl. Polym. Sci., 57, 781 (1995)

19. D. T. Grubb and K. Prasad, Macromolecules, 25, 4575 (1992).

20. D. C. Mcfaddin, K. E. Russell, G. Wu, and R. D. Heyding, $J$. Polym. Sci., Polym. Phys. Ed., 31, 175 (1993).

21. F. J. Balta-Calleja and C. G. Vonk, "X-Ray Scattering of Synthetic Polymers," Elsevier, Amsterdam, 1989.

22. R. Hosemann and W. Wilke, De Makromol. Chem., 118, 230 (1968).

23. A. M. Hindeleh, D. J. Johnson, and P. E. Montage, in "Fiber Diffraction Method," ACS Symp., No 141, A. D. French and K. H. Gardener, Ed., American Chemical Society, Washington D.C., 1983, pp 149-182.

24. X.-P. Hu, Ph. D. Thesis, The University of Leeds, United Kingdom, 1994

25. C. W. Bunn, Trans. Faraday Soc., 35, 482 (1939).

26. A. R. Stoke, Proc. Phys. Soc., A166, 283 (1938). 